\title{
Synthesis and Studies of Some New Dioxouranium (VI) Complexes with Azoester Ligands
}

\author{
Arora Kishor $^{*}{ }^{\mathrm{a}}$, Srivastava A.K. ${ }^{\mathrm{b}}$ and Chauhan A.S. ${ }^{\mathrm{b}}$ \\ ${ }^{a}$ Department of Chemistry, Govt. Postgraduate College (Autonomous); Datia (M.P.) 475 661, India \\ ${ }^{b}$ Department of Chemistry, S.M.S. Govt. Model Science College; Gwalior (M.P.) 474 001, India
}

\begin{abstract}
Some new complexes of uranyl (VI) metal ion with different anions (viz. $\mathrm{Cl}^{-}, \mathrm{I}_{\mathrm{NOO}}^{-}{ }_{3}$ and $\mathrm{OAc}^{-}$) have been synthesized using azoester ligand. Complexes were characterized by traditional methods viz. melting point measurements, conductivity measurements. These were also characterized by modern spectral methods viz. IR including far IR, UV -vis. spectra. Thermal studies specially DTA studies of representative have also been done and reported. Some of the complexes were also screened against selected microbes to check their antimicrobial activities. Coordination number of these complexes are proposed to be 8, 10 and 12 and tentative structures of the complexes are also reported in the present paper.
\end{abstract}

Keywords:-Uranyl (VI) metal complexes /azoester ligands /spectral studies/ thermal studies/ antimicrobial studies

\section{Introduction}

Metal complexes of lanthanides and that of actinides were reported with various organic compounds as ligands ${ }^{1-5}$. Schiff bases are one of the important class among these compounds which are used as ligands for this purpose.So far as the ligands are concerned, compounds which are capable of forming ring structures i.e. chelates are dominating and preferred in this area. As such complex compounds are stable, large in numbers with kinetics as well as thermodynamic stability. In fact, if more compact is the ligands and the smaller is its bite more effective it is in generating higher coordination structures.

Reactions of azo compounds have been widely studied in which nitrogen molecule is eliminated either thermally or photo chemically and two reactive sites remain react to reform a ring (Fig. 1).

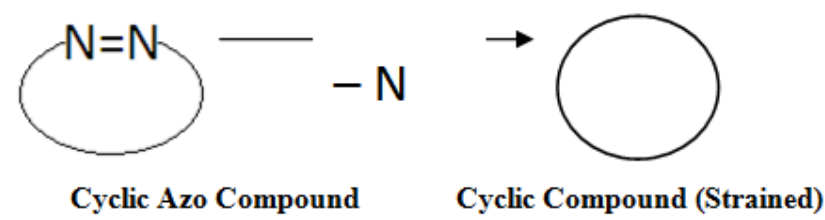

(Fig. 1)

The important synthetic application of azo compound decomposition is in synthesis of ring systems with strain. The required azo compounds can be prepared by dipolar cycloadditions of diazocompunds and the cyclic azo-esters so formed can be photochemically or thermally which finally decomposed to strained ring system. Such type of reactions have been developed for pyridazine -3, 6 - dicarboxylate esters; 1,2,4-triazines and 1,2,4,5-tetrazines etc. A large number of metal complexes ${ }^{6-10}$ of Thorium (IV) and dioxo-uranium (VI) have been reported in literature but still a possibility left to report their complexes with azoester ligands.

Keeping this in mind, these studies have been done and this present communication contains such studies related to complexes of dioxouranium(VI) metal with two azoester ligands viz. Ethyl - $\alpha$ - (3 - chloro phenylazo) acetoacetate and Ethyl - $\alpha$ - (4 - bromo phenylazo) acetoacetate.

Ligands used for formation of complexes are reported in figure 2 below.

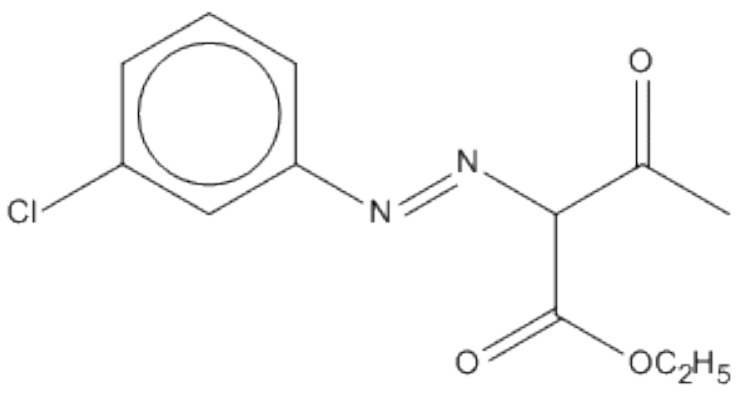




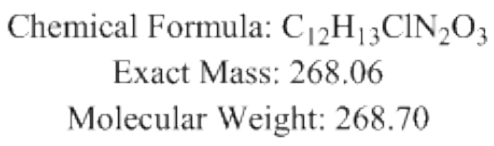

m/z: $268.06(100.0 \%), 270.06(32.1 \%), 269.06(13.7 \%), 271.06(4.5 \%), 270.07(1.4 \%)$

Elemental Analysis: C, 53.64; H, 4.88; Cl, 13.19; N, 10.43; O, 17.86

Ethyl-a-(3-chloro phenylazo) acetoacetate<smiles>CCOC(=O)C(N=Nc1ccc(Br)cc1)C(C)=O</smiles>

Chemical Formula: $\mathrm{C}_{12} \mathrm{H}_{13} \mathrm{BrN}_{2} \mathrm{O}_{3}$

Exact Mass: 312.01

Molecular Weight: 313.15

m/z: 312.01 (100.0\%), 314.01 (97.4\%), $313.01(13.7 \%), 315.01(13.5 \%), 314.02(1.4 \%)$

Elemental Analysis: C, 46.03; H, 4.18; Br, 25.52; N, 8.95; O, 15.33

Ethyl-a-(4-bromo phenylazo) acetoacetate Figure 2

\section{Experimental:}

All chemicals which are used for the purpose of preparation of new compounds were obtained in the sealed containers from standard sources e.g. Emerck, Aldrich, CDH and BDH.Uranyl (VI) metal salts viz. dioxouranium (VI) chloride, dioxouranium (VI) nitrate, dioxouranium (VI) acetate, salts were obtained from the RJ (Robbert Johson Company Ltd.) and BDH (British Drug House), Poole, England. All the chemicals were of AR, AnalR grade. Dioxouranium (VI) iodide was prepared in laboratory by reacting dioxouranium (VI) nitrate with potassium iodide.

\section{Formation of Azo-ester Ligands and their complexes}

Jolly V. S. et $\mathrm{al}^{11-12}$ have reported the synthesis of many new azo-esters. The complex forming capabilities of some of the azo-esters have been explored in the present investigations against dioxouranium(VI) metal salts. The azo-ester compounds viz. 2-Aceto-2-N (substituted phenyl) azoethylethanoates were prepared as per according to the methods reported in literature ${ }^{13-14}$. All these substituted azo-ester compounds obtained are deeply coloured i.e. reddish brown solids with low melting points and most of them became viscous in the hot summer season of this north central region of Madhya Pradesh where the temperature in this season reaches almost $44-45^{\circ} \mathrm{C}$. The reported new coordination compounds of dioxouranium (VI) metals were obtained by the reaction of the azoesters with metal salts at the $\mathrm{pH}$ ranging from 8 to 10 at the room temperature in water medium containing small amount of alcohol/butanol/mixture of two alchohols.

All the new compounds synthesisd were analysed and their analytical studies were reported as reported earlier in related literature ${ }^{6-10}$.

\section{Results And Discussions}

Analytical data of azoester ligands viz. yield, color, mol. weight etc. are reported in table 1

Table 1: Analytical Data of Azoester Ligands

\begin{tabular}{|l|l|l|l|l|l|l|}
\hline $\begin{array}{l}\text { S.No } \\
.\end{array}$ & Azoester Ligands : Structure \& Name & $\mathbf{R}$ & Yield & Color & $\begin{array}{l}\text { Molecular } \\
\text { Formula }\end{array}$ & $\begin{array}{l}\text { Molecular } \\
\text { Weight }\end{array}$ \\
\hline 1. & & &
\end{tabular}




\begin{tabular}{|l|l|l|l|l|l|l|}
\hline & $\begin{array}{l}\text { Ethyl }-\alpha-(3-\text { chloro phenylazo }) \\
\text { acetoacetate }\end{array}$ & & & & \\
\hline 2. & $\begin{array}{l}\text { Ethyl }-\alpha-\mathrm{Br} \\
\text { acetoacetate }\end{array}$ & $80 \%$ & $\begin{array}{l}\text { Light } \\
\text { Brown }\end{array}$ & $\mathrm{C}_{12} \mathrm{H}_{13} \mathrm{BrN}_{2} \mathrm{O}_{3}$ & 286 \\
\hline
\end{tabular}

Mass spectral data of these azoester compounds i.e. ligands are also reported in the tables 2,3 and the prominent peaks observed are listed in these tables for these compounds. Base ion peak are marked and shown in the tables for these compounds. Though spectra of azosters show the peaks related to fragments that may be formed after fragmentation but investigator was not able to record parent ion peak.

Table 2 :Mass Spectral Details of Ethyl $-\alpha-(3-$ chloro phenylazo) acetoacetate

\begin{tabular}{|l|l|}
\hline $\mathbf{m} / \mathbf{z}$ & Relative Abundance (\%) \\
\hline 206 & 5 \\
\hline 127 & 99 \\
\hline 112 & 10 \\
\hline 102 & 15 \\
\hline 92 & 20 \\
\hline 75 & 2 \\
\hline 65 & 35 \\
\hline 52 & 10 \\
\hline
\end{tabular}

Table 3 :Mass Spectral Details of Ethyl $-\alpha-(4-$ bromo phenylazo) acetoacetate $)$

\begin{tabular}{|l|l|}
\hline $\mathbf{m} / \mathbf{z}$ & Relative Abundance (\%) \\
\hline 207 & 2 \\
\hline 156 & 45 \\
\hline 143 & 2 \\
\hline 129 & 2 \\
\hline 117 & 2 \\
\hline 104 & 2 \\
\hline 77 & 95 \\
\hline 62 & 2 \\
\hline 51 & 32 \\
\hline
\end{tabular}

Despite of the semi solid physical state of the azo ester ligands during summer season the new complexes of uranyl (VI) metal ion obtained were all crystalline solid with generally high melting points. These were stored in polythene tubes after re-crystallization. The purity of the new compounds was checked by the chromatographic methods. The new complexes prepared are listed below and their analytical data are presented in table 4.

1. [Bis (ethyl- $\alpha-(3-$ chloro phenylazo) acetoacetato) diiodo] Dioxouranium (VI)

2. [Bis (ethyl- $\alpha-(3-$ chloro phenylazo) acetoacetato) dinitro] Dioxouranium (VI)

3. [Bis (ethyl- $\alpha-(3-$ chloro phenylazo) acetoacetato) dichloro] Dioxouranium (VI)

4. [Bis (ethyl- $\alpha-(3-$ chloro phenylazo) acetoacetato) diacetato] Dioxouranium (VI)

5. [Bis (ethyl- $\alpha-(4-$ bromo phenylazo) acetoacetato) diiodo] Dioxouranium (VI)

6. [Bis (ethyl- $\alpha-(4-$ bromo phenylazo) acetoacetato) diacetato] Dioxouranium (VI)

7. [Bis (ethyl- $\alpha-(4-$ bromo phenylazo) acetoacetato) dinitro] Dioxouranium (VI)

Table 4: Analytical Data of The New Metal Complexes of Dioxouranium (VI) Metal Salts with Substituted Azoester Ligands

\begin{tabular}{|l|l|l|l|l|}
\hline S. No. & Compound Name & Molecular Formula & Color & $\begin{array}{l}\text { Molecular } \\
\text { Weight } \\
\text { Calculated } \\
\text { (Observed) }\end{array}$ \\
\hline 1. & $\begin{array}{l}\text { [Bis (ethyl- } \alpha-(3-\text { chloro phenylazo) } \\
\text { acetoacetato) diiodo] Dioxouranium (VI) }\end{array}$ & $\mathrm{C}_{24} \mathrm{H}_{28} \mathrm{I}_{2} \mathrm{~N}_{4} \mathrm{O}_{8} \mathrm{U}$ & $\begin{array}{l}992.34 \\
(985)\end{array}$ \\
\hline 2. & $\begin{array}{l}\text { [Bis (ethyl- } \alpha-(3-\text { chloro phenylazo) } \\
\text { acetoacetato] dinitro] Dioxouranium (VI) }\end{array}$ & $\mathrm{C}_{24} \mathrm{H}_{26} \mathrm{Cl}_{2} \mathrm{~N}_{6} \mathrm{O}_{14} \mathrm{U}$ & $\begin{array}{l}\text { Deep } \\
\text { Yellow }\end{array}$ & $\begin{array}{l}931.43 \\
(927)\end{array}$ \\
\hline 3. & $\begin{array}{l}\text { [Bis (ethyl- } \alpha-(3-\text { chloro phenylazo) } \\
\text { acetoacetato) diacetato] Dioxouranium (VI) }\end{array}$ & $\mathrm{C}_{28} \mathrm{H}_{32} \mathrm{Cl}_{2} \mathrm{~N}_{4} \mathrm{O}_{12} \mathrm{U}$ & $\begin{array}{l}\text { Light } \\
\text { Yellow }\end{array}$ & $\begin{array}{l}925.51 \\
(918)\end{array}$ \\
\hline 4. & $\begin{array}{l}\text { [Bis (ethyl- } \alpha-(3-\text { chloro phenylazo) } \\
\text { acetoacetato) dichloro] Dioxouranium (VI) }\end{array}$ & $\mathrm{C}_{24} \mathrm{H}_{26} \mathrm{Cl}_{4} \mathrm{~N}_{4} \mathrm{O}_{8} \mathrm{U}$ & $\begin{array}{l}\text { Light } \\
\text { Yellow }\end{array}$ & $\begin{array}{l}878.33 \\
(870)\end{array}$ \\
\hline
\end{tabular}


Synthesis and Studies of Some New Dioxouranium (VI) Complexes with Azoester Ligands

\begin{tabular}{|l|l|l|l|l|}
\hline 5. & $\begin{array}{l}{[\text { Bis (ethyl- } \alpha-(4-\text { bromo phenylazo }} \\
\text { acetoacetato) diiodo] Dioxouranium (VI) }\end{array}$ & $\mathrm{C}_{24} \mathrm{H}_{26} \mathrm{Br}_{2} \mathrm{I}_{2} \mathrm{~N}_{4} \mathrm{O}_{8} \mathrm{U}$ & Brown & $\begin{array}{l}1150.13 \\
(1145)\end{array}$ \\
\hline 6. & $\begin{array}{l}\text { Bis (ethyl- } \alpha-(4-\text { bromo phenylazo) } \\
\text { acetoacetato) dinitro] Dioxouranium (VI) }\end{array}$ & $\mathrm{C}_{24} \mathrm{H}_{26} \mathrm{Br}_{2} \mathrm{~N}_{6} \mathrm{O}_{14} \mathrm{U}$ & Brown & $\begin{array}{l}1020.33 \\
(1015)\end{array}$ \\
\hline 7. & $\begin{array}{l}\text { Bis (ethyl- } \alpha-(4-\text { bromo phenylazo) } \\
\text { acetoacetato) diacetato] Dioxouranium (VI) }\end{array}$ & $\mathrm{C}_{29} \mathrm{H}_{35} \mathrm{Br}_{2} \mathrm{~N}_{4} \mathrm{O}_{12} \mathrm{U}$ & Brown & $\begin{array}{l}1029.44 \\
(1020)\end{array}$ \\
\hline
\end{tabular}

All of these complexes are insoluble in common organic solvents. They were sparingly soluble in DMF and DMSO. Conductivity measurements in DMF for these complexes were too low to account for any disassociation of these complexes in DMF at the concentration of the $10^{-5} \mathrm{M}$. Hence, these complexes may be regarded as non electrolytes.

\section{IR spectral studies}

IR spectral analysis of the azoester ligands used for complex formation along with that of the representative complexes are reported in the tables 5-10. The $\mathrm{N}=\mathrm{N}$ stretching vibration ${ }^{15}$ of a symmetrical transazo compound is forbidden in the infra red spectrum but absorbs and appears ca $1576 \mathrm{~cm}^{-1}$ region of the Raman spectrum. Unsymmetrical para-substituted azo-benzenes in which the substituent is an electron donating group absorbs ca $1429 \mathrm{~cm}^{-1}$. The bands are weak because of the non-polar nature of the bond. The bands at $1570 \mathrm{~cm}^{-1}$ and $1590 \mathrm{~cm}^{-1}$ due to $\mathrm{v}(\mathrm{N}=\mathrm{N})$ undergo batho-chromic shift to $1550 \mathrm{~cm}^{-1}$ in metal complexes clearly indicates that one of the azo nitrogen is bonded to the metal atom. ${ }^{16-17}$

Table 5 : IR Absorption frequency $\left(\mathrm{cm}^{-1}\right)$ of [Bis (Ethyl- $\alpha-(3-$ Chloro phenylazo acetoacetato) dinitro) dioxouranium $(\mathrm{VI})$

\begin{tabular}{|l|l|l|}
\hline Assignment & Ligand & Complex \\
\hline Aromatic C $-\mathrm{H}$ stretching & 3200 & 3350 \\
\hline $\mathrm{C}=$ O Stretching ester & - & - \\
\hline $\mathrm{C}-\mathrm{N}$ Stretching & 1425 & 1580 \\
\hline \\
\hline
\end{tabular}

Table 6: IR Absorption frequency $\left(\mathrm{cm}^{-1}\right)$ of [Bis (Ethyl- $\alpha-(3-$ Chloro phenylazo acetoacetato) diacetato) dioxouranium $(\mathrm{VI})$

\begin{tabular}{|l|l|l|}
\hline Assignment & Ligand & Complex \\
\hline Aromatic CH stretching & 3100 & 3350 \\
\hline $\mathrm{C}=$ O Stretching ester & 1700 & 1700 \\
\hline $\mathrm{C}-\mathrm{N}$ Stretching & 1425 & 1500 \\
\hline $\mathrm{C}-\mathrm{Cl}$ Stretching & 1100 & 1080 \\
\hline Out of place C $-\mathrm{H}$ vibration & 900 & 910 \\
\hline Out of place C $-\mathrm{C}$ Bending & 700 & 690 \\
\hline $\mathrm{M}-\mathrm{N}$ vibration & - & 490 \\
\hline
\end{tabular}

Table 7 : IR Absorption frequency $\left(\mathrm{cm}^{-1}\right)$ of [Bis (Ethyl- $\alpha-(3-$ Chloro phenylazo acetoacetato) dichloro) dioxouranium (VI)

\begin{tabular}{|l|l|l|}
\hline Assignment & Ligand & Complex \\
\hline Aromatic C - H stretching & 3100 & 3300 \\
\hline C = O Stretching ester & 1720 & 1760 \\
\hline C-N Stretching & 1425 & 1500 \\
\hline O & & \\
II & 1350 & 1400 \\
\hline
\end{tabular}




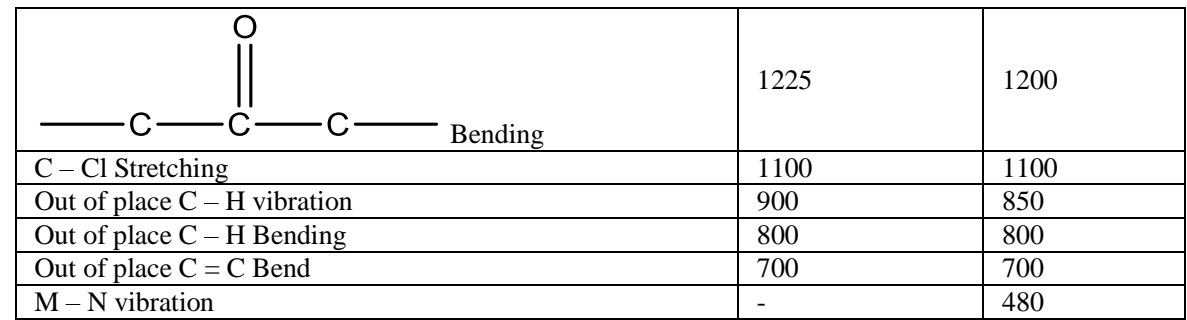

Table 8 : IR Absorption frequency $\left(\mathrm{cm}^{-1}\right)$ of [Bis (Ethyl- $\alpha-(4-$ bromo phenylazo acetoacetato) diiodo) dioxouranium $(\mathrm{VI})$

\begin{tabular}{|l|l|l|}
\hline Assignment & Ligand & Complex \\
\hline Aromatic CH stretching & 3200 & 3400 \\
\hline C $=$ O stretching in ester & 1750 & 1600 \\
\hline C $=$ C stretching & 1520 & 1520 \\
\hline C $=$ O stretching in ester & 1350 & 1390 \\
\hline Disubstituted Benzene & 1100 & 1080 \\
\hline C - H deformation for disubstituted benzene & 800 & 800 \\
\hline C - Br stretching & 550 & 600 \\
\hline M - N vibration & - & 480 \\
\hline
\end{tabular}

Table 9 : IR Absorption frequency $\left(\mathrm{cm}^{-1}\right)$ of [Bis (Ethyl- $\alpha-(4-$ bromo phenylazo acetoacetato) diacetato) dioxouranium (VI)

\begin{tabular}{|l|l|l|}
\hline Assignment & Ligand & Complex \\
\hline Aromatic CH stretching & 3200 & 3250 \\
\hline $\mathrm{C}=\mathrm{O}$ stretching in ester & 1750 & 1600 \\
\hline $\mathrm{C}=\mathrm{C}$ stretching & 1520 & 1510 \\
\hline $\mathrm{C}=\mathrm{O}$ stretching in ester & 1350 & 1310 \\
\hline Disubstituted Benzene & 1100 & 1100 \\
\hline $\mathrm{C}-\mathrm{H}$ deformation for disubstituted benzene & 800 & 810 \\
\hline $\mathrm{C}-$ Br stretching & 550 & 520 \\
\hline $\mathrm{M}-\mathrm{N}$ vibrational & - & 480 \\
\hline
\end{tabular}

Table 10 : IR Absorption frequency $\left(\mathrm{cm}^{-1}\right)$ of [Bis (Ethyl- $\alpha-(4-$ bromo phenylazo acetoacetato) dinitro) dioxouranium (VI) Solvent $\mathrm{C}_{2} \mathrm{H}_{5} \mathrm{OH}$

\begin{tabular}{|l|l|l|}
\hline Assignment & Ligand & Complex \\
\hline Aromatic C $-\mathrm{H}$ stretching & 3200 & 3450 \\
\hline $\mathrm{C}=\mathrm{O}$ stretching in ester & 1750 & 1600 \\
\hline $\mathrm{C}=\mathrm{C}$ stretching & 1520 & 1550 \\
\hline $\mathrm{C}=\mathrm{O}$ stretching in ester & 1350 & 1390 \\
\hline Disubstituted Benzene & 1100 & 1100 \\
\hline $\mathrm{C}-\mathrm{H}$ deformation for disubstituted benzene & 800 & 850 \\
\hline $\mathrm{C}-$ Br stretching & 550 & 550 \\
\hline $\mathrm{M}-\mathrm{N}$ vibration & - & 480 \\
\hline
\end{tabular}

Table 11: IR Absorption frequency $\left(\mathrm{cm}^{-1}\right)$ of [Bis (Ethyl- $\alpha-(4-$ bromo phenylazo acetoacetato) dinitro) dioxouranium (VI) Solvent $\mathrm{C}_{2} \mathrm{H}_{5} \mathrm{OH}+\mathrm{KOH}$

\begin{tabular}{|l|l|l|}
\hline Assignment & Ligand & Complexes \\
\hline Aromatic C - M stretching & 3200 & 3450 \\
\hline $\mathrm{C}=\mathrm{O}$ stretching in ester & 1750 & 1760 \\
\hline $\mathrm{C}=\mathrm{C}$ stretching & 1520 & 1520 \\
\hline $\mathrm{C}=\mathrm{O}$ stretching in ester & 1350 & 1400 \\
\hline Disubstituted Benzene & 1100 & 1100 \\
\hline $\mathrm{C}-\mathrm{H}$ deformation for disubstituted benzene & 800 & 900 \\
\hline $\mathrm{C}-$ Br stretching & 550 & 760 \\
\hline $\mathrm{M}-\mathrm{N}$ vibration & - & 490 \\
\hline
\end{tabular}

Infrared (IR) spectroscopy has also been proved to be a tool to establish anions present in the

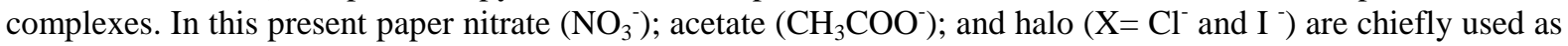
anionic counter part to metal ions in the complexes under investigation. The IR studies of nitrato ions in these complexes under studies are reported in table 12 below. 
Table 12: Infrared Absorption frequencies $\left(\mathrm{cm}^{-1}\right)$ of Nitrato Groups of dioxouranium (VI) metal Complexes of Azoester Ligands

\begin{tabular}{|c|c|c|c|c|c|c|c|}
\hline Complexes & $v_{4}$ & $v_{1}$ & $v_{2}$ & $v_{6}$ & $v_{3}$ & $U_{5}$ & Ref. \\
\hline $\mathrm{UO}_{2}\left(\mathrm{NO}_{3}\right)_{2} \cdot 2(\mathrm{MeP})$ & $1510 \mathrm{sh}$ & $1310 \mathrm{~s}$ & $1040 \mathrm{~m}$ & $805 \mathrm{sh}$ & $742 \mathrm{~m}$ & $705 \mathrm{~m}$ & 8,9 \\
\hline $\mathrm{UO}_{2}\left(\mathrm{NO}_{3}\right)_{2} .2(\mathrm{MPP})$ & $1500 \mathrm{~s}$ & $1280 \mathrm{w}$ & $1025 \mathrm{~s}$ & $820 \mathrm{~m}$ & $755 \mathrm{~s}$ & - & 8,9 \\
\hline $\mathrm{UO}_{2}\left(\mathrm{NO}_{3}\right)_{2} .2(\mathrm{MNP})$ & $1500 \mathrm{sh}$ & $1310 \mathrm{~m}$ & $1040 \mathrm{~m}$ & $805 \mathrm{~m}$ & $742 \mathrm{w}$ & $705 \mathrm{w}$ & 8,9 \\
\hline $\begin{array}{lr}\text { Bis } & \text { (Ethyl- } \alpha-(3-\text { chloro } \\
\text { phenylazo } & \text { acetoacetato) } \\
\text { dinitro) Dioxouranium (VI) }\end{array}$ & $1500 \mathrm{sh}$ & $1310 \mathrm{sh}$ & $1040 \mathrm{~m}$ & $820 \mathrm{~m}$ & $750 \mathrm{w}$ & $710 \mathrm{w}$ & $\begin{array}{l}\text { This } \\
\text { work }\end{array}$ \\
\hline $\begin{array}{lr}\text { Bis } & \text { (Ethyl- } \alpha-(4-\text { bromo } \\
\text { phenylazo } & \text { acetoacetato) } \\
\text { dinitro) Dioxouranium (VI) }\end{array}$ & $1510 \mathrm{~s}$ & $1300 \mathrm{sh}$ & $1050 \mathrm{w}$ & $825 \mathrm{w}$ & $760 \mathrm{~m}$ & $710 \mathrm{w}$ & $\begin{array}{l}\text { This } \\
\text { work }\end{array}$ \\
\hline
\end{tabular}

Lever separation rule may be applied to distinguish between mono-dentate or bi-dentate nitrate ions. Lever et. $\mathrm{al}^{18}$ showed that this to be true that the separation for mono-dentate nitrate in $\left(v_{1}-v_{4}\right)$ is appeared to be $5-26 \mathrm{~cm}^{-1}$ and that for bi-dentate nitrate group this may lies in $20-60 \mathrm{~cm}^{-1}$. This method has been tried in present complexes and a separation of $\mathrm{Ca} 250-200 \mathrm{~cm}^{-1}$ in the combination bands in the region $1550-1290$ $\mathrm{cm}^{-1}$ has been investigated which suggest that nitrate ion may be bi-dentate in nature in these complexes ${ }^{19-21}$. Similar to nitrate, acetate ion in the present study of complexes also appears to be bi-dentate ligand. In the case of halo complexes (M-X) IR spectral vibrations could not be assigned because they are out of the range of studied region used in the present investigations.

\section{Oxocation $(\mathrm{O}=\mathrm{U}=\mathrm{O})$ Vibrations:}

Uranyl (VI or dioxouranium (VI) ion is quite peculiar both in its own structure and in its coordination behavior. This ions retains its identity over a wide range of vibrations in experimental conditions and can be considered as a single particle from geometric point of view. It has four fundamental vibrations viz.

(i) Non-degenerate symmetric stretching vibration $v_{2}$ (normally IR forbidden $~ 900-800 \mathrm{~cm}^{-1}$ )

(ii) Non-degenerate symmetric stretching vibration $v_{3}$ (usually IR active $\sim 1000-900 \mathrm{~cm}^{-1}$ ) and

(iii) Doubly degenerate O-U-O bending vibration $v_{2}$ (IR active $\sim 750-700 \mathrm{~cm}^{-1}$ )

In the present investigation the $\mathrm{U}=\mathrm{O}$ in all the cases has been assigned $v_{3}$ and $v_{1}$ approximately in the region $920-890 \mathrm{~cm}^{-1}$ and $850-800 \mathrm{~cm}^{-1}$ respectively as recorded and shown in table 13.

Table 13: Oxocation $(\mathrm{O}=\mathrm{U}=\mathrm{O})$ Related Vibrations $\mathrm{v}_{1}$ and $\mathrm{v}_{3}(\mathrm{~cm}-1)$ for Dioxouranium (VI) Complexes of Azoester Ligands

\begin{tabular}{|c|c|c|}
\hline Complexes & $v_{1}$ & $U_{3}$ \\
\hline [Bis (Ethyl- $\alpha$-(4-bromo phenylazo) acetoacetato) diacetato] dioxouranium (VI) & $810 \mathrm{w}$ & $920 \mathrm{~s}$ \\
\hline [Bis (Ethyl- $\alpha$-(3-chloro phenylazo) acetoacetato) diacetato] dioxouranium (VI) & $820 \mathrm{sh}$ & $910 \mathrm{~s}$ \\
\hline [Bis (Ethyl- $\alpha$-(3-chloro phenylazo) acetoacetato) dinitro] dioxouranium (VI) & $825 \mathrm{w}$ & $890 \mathrm{~s}$ \\
\hline $\begin{array}{l}\text { [Bis (Ethyl- } \alpha \text {-(4-bromo phenylazo) acetoacetato) dinitro] dioxouranium (VI) Solvent } \\
\mathrm{C}_{2} \mathrm{H}_{5} \mathrm{OH}+\mathrm{KOH}\end{array}$ & $825 \mathrm{w}$ & $900 \mathrm{~s}$ \\
\hline $\begin{array}{l}\text { [Bis (Ethyl- } \alpha \text {-(4-bromo phenylazo) acetoacetato) dinitro] dioxouranium (VI) Solvent } \\
\mathrm{C}_{2} \mathrm{H}_{5} \mathrm{OH}\end{array}$ & $850 \mathrm{~m}$ & $900 \mathrm{sh}$ \\
\hline [Bis (Ethyl- $\alpha$-(3-chloro phenylazo) acetoacetato) dichloro] dioxouranium (VI) & $850 \mathrm{~m}$ & $900 \mathrm{sh}$ \\
\hline [Bis (Ethyl- $\alpha$-(3-chloro phenylazo) acetoacetato) diiodo] dioxouranium (VI) & $810 \mathrm{w}$ & $890 \mathrm{~s}$ \\
\hline [Bis (Ethyl- $\alpha$-(4-bromo phenylazo) acetoacetato) diiodo] dioxouranium (VI) & $800 \mathrm{~s}$ & $920 \mathrm{~s}$ \\
\hline
\end{tabular}

\section{UV- Visible spectral studies}

UV- Visible spectral analysis of some of the representative complexes have also been done and these are reported in tables 14,15 below

Table 14 : UV-Visible Spectra of Ethyl- $\alpha-(3-$ Chlorophenyl azo aceto acetato) and Various Complexes with Dioxouranium (VI) Transition (nm)

\begin{tabular}{|l|l|l|l|}
\hline S. No. & Ligands/Complex & $\boldsymbol{\pi}-\boldsymbol{\pi}^{*}$ & $\boldsymbol{\pi}-\boldsymbol{\pi}^{*}$ \\
\hline 1. & Ethyl $-\alpha-(3-$ Chloro phenylazo) acetoacetate & 402 & 487 \\
\hline 2. & {$[$ Bis (Ethyl $-\alpha-(3-$ Chloro phenylazo) acetoacetato) diiodo] dioxouranium (VI) } & 340 & 360 \\
\hline 3. & {$[$ Bis (Ethyl $-\alpha-(3-$ Chloro phenylazo) acetoacetato) dinitro] dioxouranium (VI) } & 340 & 360 \\
\hline 4. & {$[$ Bis (Ethyl- $\alpha-(3-$ Chloro phenylazo) acetoacetato) diaceteto] dioxouranium (VI) } & 450 & 460 \\
\hline 5. & {$[$ Bis (Ethyl $-\alpha-(3-$ Chloro phenyl azo) acetoacetato) dichloro] dioxouranium (VI) } & 450 & 460 \\
\hline
\end{tabular}

Table 15 : UV-Visible Spectra of Ethyl- $\alpha$-(4-bromo phenylazo) aceto acetato) and Various Complexes with Dioxouranium (VI) Transition (nm)

\begin{tabular}{|c|c|c|c|}
\hline S. No. & Ligands/Complex & $n \rightarrow \pi^{*}$ & $\pi-\pi^{*}$ \\
\hline 1. & Ethyl $-\alpha-(4$-bromo phenylazo) acetoacetate & 374 & 437 \\
\hline
\end{tabular}


Synthesis and Studies of Some New Dioxouranium (VI) Complexes with Azoester Ligands

\begin{tabular}{|l|l|l|}
\hline 3. & {$[$ Bis (Ethyl $-\alpha-(4-$ bromo phenylazo) acetoacetato) diacetato] dioxouranium (VI) } & 360 \\
\hline 4. & $\begin{array}{l}{[\text { Bis (Ethyl }-\alpha-(4-\text { bromo phenylazo) acetoacetato) dinitro] dioxouranium (VI) solvent }} \\
\mathrm{C}_{2} \mathrm{H}_{5} \mathrm{OH}\end{array}$ & 320 \\
\hline 5. & $\begin{array}{l}{[\mathrm{Bis}(\text { Ethyl }-\alpha-(4-\text { bromo phenylazo) acetoacetato) dinitro] dioxouranium (VI) solvent }} \\
\mathrm{C}_{2} \mathrm{H}_{5} \mathrm{OH}+\mathrm{KOH}\end{array}$ & 400 \\
\hline
\end{tabular}

\section{Magnetic Behaviour of Complexes:}

Complexes of uranyl (VI) metal salts are diamagnetic in nature depending upon the other ions present and the corresponding ligand field. The magnetic susceptibilities are independent of field strength and temperature. In the present studies complexes are weakly diamagnetic as observed ${ }^{22-24}$.

\section{DTA studies of the Complexes}

The results of these Differential Thermal Analysis (DTA) studies which are carried out on representative complexes under studies are reported in Table 13 -14. The observed DTA graphs clearly show the stability of complexes up to considerably higher temperature range. The peaks located as EXO and ENDO peaks are mentioned in tables below. In all of these cases oxides are formed as final products of analysis viz. stable $\mathrm{ThO}_{2}$ and $\mathrm{U}_{3} \mathrm{O}_{8}$ over a comparatively high range of temperatures. These studies confirm the high stability of these complexes under studies up to high temperature. In intermediate steps of DTA analysis it has been suggested that ligand moiety may left the complex molecules. Results of DTA are presented in tables 16 and 17.

Table 16 : DTA Study of [Bis (Ethyl- $\alpha$-(3-chloro phenylazo) acetoacetato) diacetato] Dioxouranium (VI) $\left(\mathrm{C}_{28} \mathrm{H}_{32} \mathrm{Cl}_{2} \mathrm{~N}_{4} \mathrm{O}_{12} \mathrm{U}\right)$, Mol. Wt. - 924.19

\begin{tabular}{|l|l|l|l|}
\hline S. No. & Temperature Range $\left({ }^{\circ} \mathbf{C}\right)$ & Peaks & Final Product \\
\hline 1. & $110^{\circ}-120^{\circ}$ & Endo & - \\
\hline 2. & $200^{\circ}-210^{\circ}$ & Exo & - \\
\hline 3. & $340^{\circ}-$ Above & Exo & $\mathrm{U}_{3} \mathrm{O}_{8}$ \\
\hline
\end{tabular}

Table 17: DTA Study of [Bis (Ethyl- $\alpha$-(4-bromo phenylazo) acetoacetato) dinitro] Dioxouranium (VI) $\left(\mathrm{C}_{24} \mathrm{H}_{26} \mathrm{Br}_{2} \mathrm{~N}_{6} \mathrm{O}_{14} \mathrm{U}\right)$, Mol. Wt. - 1020.33

\begin{tabular}{|l|l|l|l|}
\hline S. No. & Temperature Range $\left({ }^{\circ} \mathbf{C}\right)$ & Peaks & Final Product \\
\hline 1. & $40^{\circ}-50^{\circ}$ & Endo & - \\
\hline 2. & $150^{\circ}-180^{\circ}$ & Exo & - \\
\hline 3. & $300^{\circ}-325^{\circ}$ & Exo & $\mathrm{U}_{3} \mathrm{O}_{8}$ \\
\hline
\end{tabular}

Based on the studies done on newly synthesized complexes of uranyl(VI) metal salts, their tentative structures were proposed along with their coordination numberswhich are given in figure 3 below.

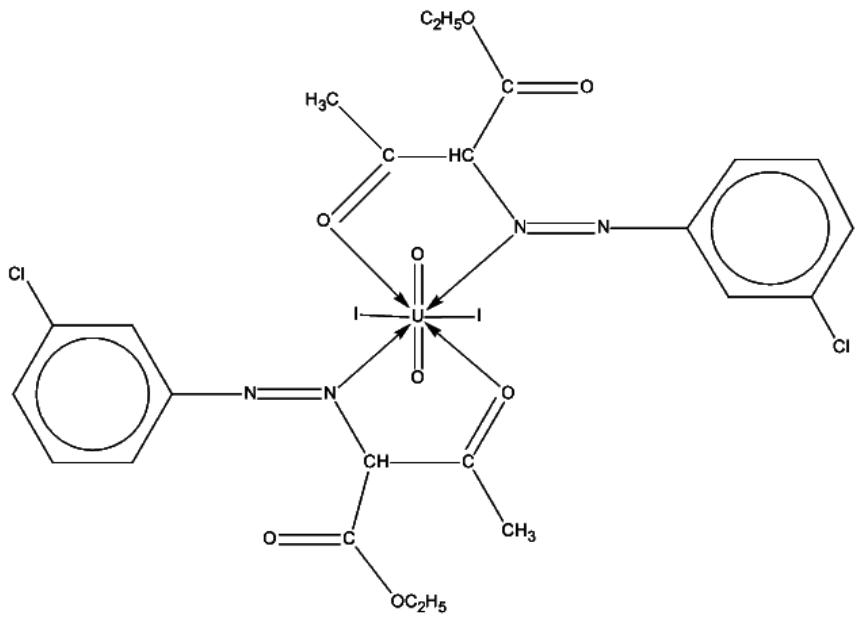

$$
\text { C.N. }=8
$$

Chemical Formula: $\mathrm{C}_{24} \mathrm{H}_{26} \mathrm{Cl}_{2} \mathrm{I}_{2} \mathrm{~N}_{4} \mathrm{O}_{8} \mathrm{U}$

Exact Mass: 1059.97

Molecular Weight: 1061.23

m/z: $1059.97(100.0 \%), 1061.97(64.2 \%), 1060.98(26.5 \%), 1062.97(17.7 \%), 1063.97(11.5 \%), 1061.98$ $(5.0 \%), 1064.97(2.8 \%), 1063.98(2.2 \%), 1060.97(1.5 \%)$

Elemental Analysis: C, 27.16; H, 2.47; Cl, 6.68; I, 23.92; N, 5.28; O, 12.06; U, 22.43

Bis (Ethyl-a(3-chlorophenylazo acetoacetato) diiodo dioxouranium(VI) 


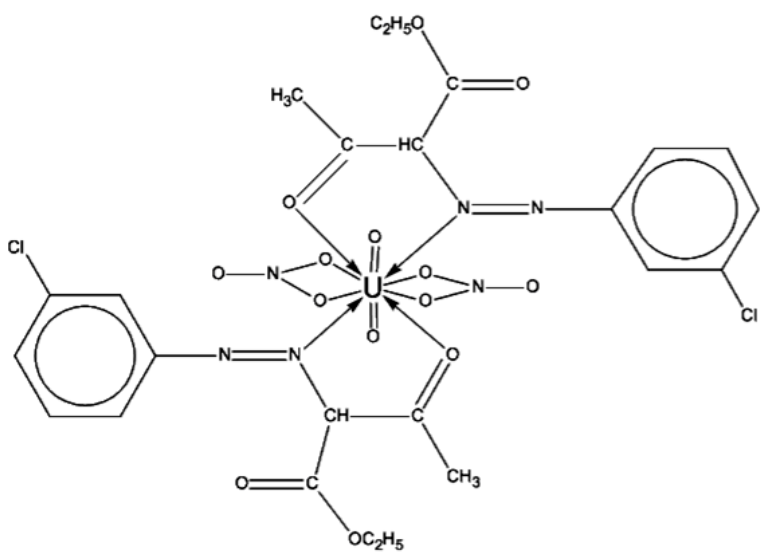

$$
\text { C.N. }=10
$$

Chemical Formula: $\mathrm{C}_{24} \mathrm{H}_{26} \mathrm{Cl}_{2} \mathrm{~N}_{6} \mathrm{O}_{14} \mathrm{U}$

Exact Mass: 930.14

Molecular Weight: 931.43

m/z: $930.14(100.0 \%), 932.14(67.4 \%), 931.14(28.7 \%), 933.14(17.3 \%), 934.13(10.2 \%), 934.14(4.4 \%)$, $932.15(3.4 \%), 935.14(3.3 \%), 933.13(1.4 \%), 933.15(1.1 \%)$

Elemental Analysis: C, 30.95; H, 2.81; Cl, 7.61; N, 9.02; O

\section{Bis (Ethyl- $\alpha$ (3-chlorophenylazo acetoacetato) dinitro dioxouranium( VI)}

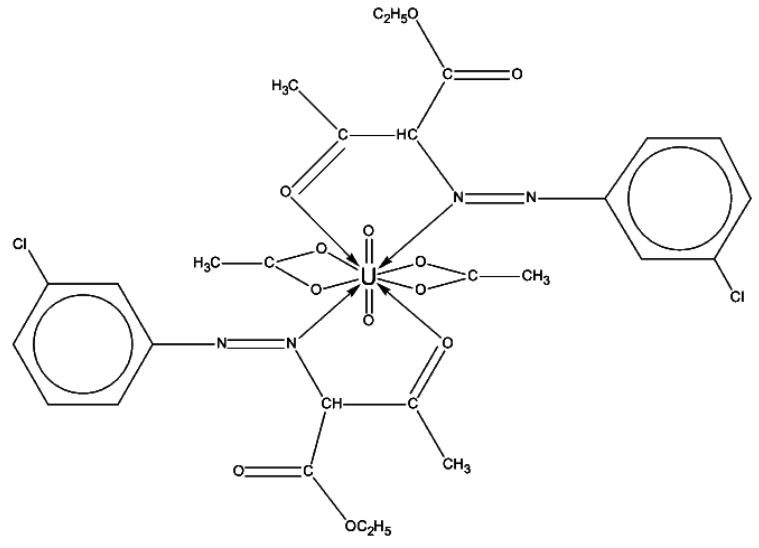

$$
\text { C.N. }=10
$$

Chemical Formula: $\mathrm{C}_{28} \mathrm{H}_{32} \mathrm{Cl}_{2} \mathrm{~N}_{4} \mathrm{O}_{12} \mathrm{U}$

Exact Mass: 924.19

Molecular Weight: 925.51

m/z: $924.19(100.0 \%), 926.19(66.8 \%), 925.19(32.2 \%), 927.19(20.0 \%), 928.18(10.2 \%), 928.19(4.8 \%)$, $926.20(4.7 \%), 929.19(3.7 \%), 927.20(1.2 \%)$

Elemental Analysis: C, 36.34; H, 3.49; Cl, 7.66; N, 6.05; O, 20.74; U, 25.72

Bis (Ethyl-a(3-chlorophenylazo acetoacetato) diacetato dioxouranium(VI)

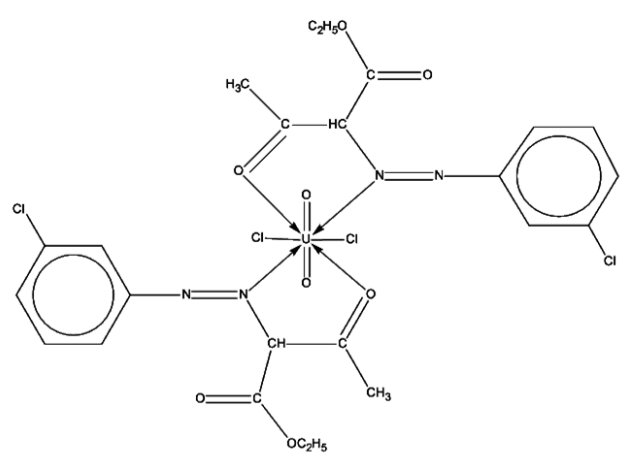




$$
\text { C.N. }=8
$$

Chemical Formula: $\mathrm{C}_{24} \mathrm{H}_{26} \mathrm{Cl}_{4} \mathrm{~N}_{4} \mathrm{O}_{8} \mathrm{U}$

Exact Mass: 876.10

Molecular Weight: 878.33

$\mathrm{m} / \mathrm{z}: 878.10(100.0 \%), 876.10(78.0 \%), 880.10(49.8 \%), 879.10(28.0 \%), 877.10(21.4 \%), 881.10(12.8 \%)$, $882.09(10.2 \%), 878.11(3.9 \%), 880.11(3.4 \%), 883.10(2.9 \%), 882.10(2.6 \%)$

Elemental Analysis: C, 32.82; H, 2.98; Cl, 16.15; N, 6.38; O, 14.57; U, 27.10

[Bis(Ethyl- $\alpha$ (3-chlorophenylazo acetoacetato) dichloro] dioxouranium (VI)

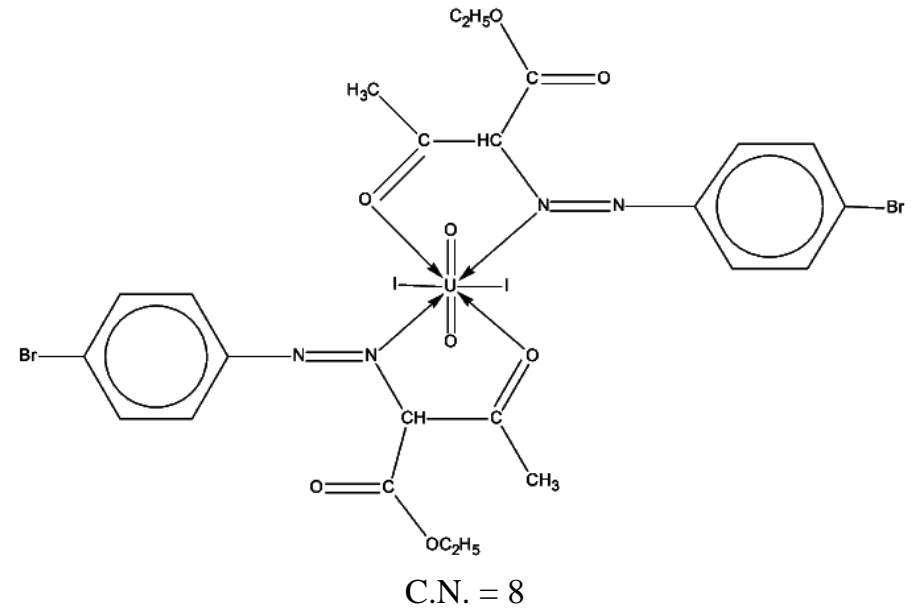

Chemical Formula: $\mathrm{C}_{24} \mathrm{H}_{26} \mathrm{Br}_{2} \mathrm{I}_{2} \mathrm{~N}_{4} \mathrm{O}_{8} \mathrm{U}$

Exact Mass: 1147.87

Molecular Weight: 1150.13

$\mathrm{m} / \mathrm{z}: 1149.87(100.0 \%), 1147.87(51.5 \%), 1151.87(50.6 \%), 1150.87(27.7 \%), 1148.87(14.1 \%), 1152.87$

$(13.0 \%), 1151.88(3.4 \%), 1153.87(2.6 \%), 1149.88(2.6 \%)$

Elemental Analysis: C, 25.06; H, 2.28; Br, 13.89; I, 22.07; N, 4.87; O, 11.13; U, 20.70

[Bis(Ethyl-a(4-bromophenylazo acetoacetato) diiodo] dioxouranium (VI)

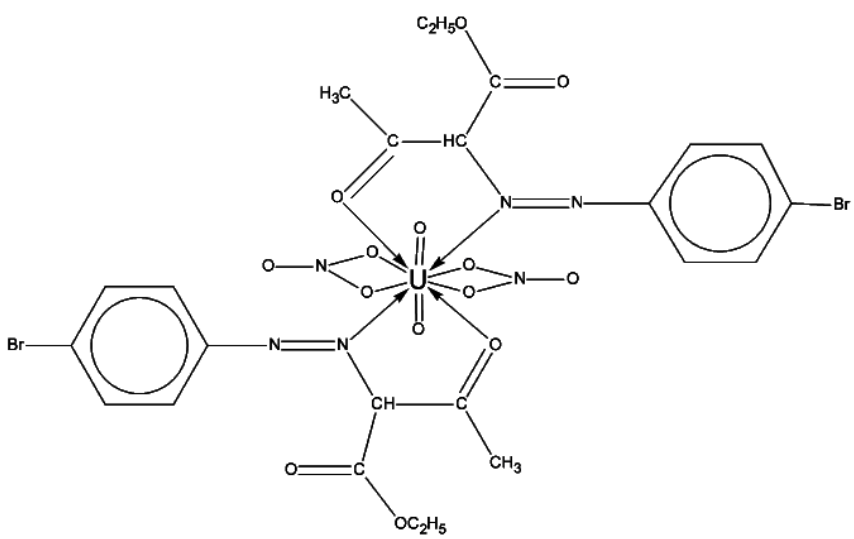

Chemical Formula: $\mathrm{C}_{24} \mathrm{H}_{26} \mathrm{Br}_{2} \mathrm{~N}_{6} \mathrm{O}_{14} \mathrm{U}$

Exact Mass: 1018.04

Molecular Weight: 1020.33

m/z: $1020.04(100.0 \%), 1018.04(49.7 \%), 1022.03(47.0 \%), 1021.04(26.0 \%), 1019.04(14.4 \%), 1023.04$

$(13.5 \%), 1022.04(6.6 \%), 1024.04(3.0 \%), 1021.03(2.2 \%), 1023.03(1.0 \%)$

Elemental Analysis: C, $28.25 ; \mathrm{H}, 2.57$; Br, 15.66; N, 8.24; O, 21.95; U, 23.33

$$
\text { C.N. }=10
$$

[Bis (Ethyl-a(4-bromo phenylazo aceto acetato) dinitro] dioxouranium (VI) 


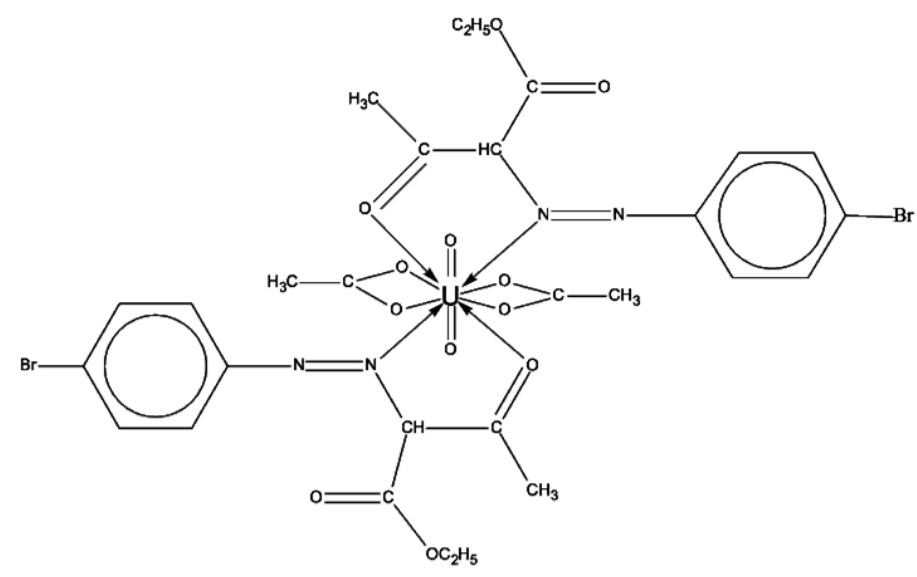

$$
\text { C.N. }=10
$$

Chemical Formula: $\mathrm{C}_{29} \mathrm{H}_{35} \mathrm{Br}_{2} \mathrm{~N}_{4} \mathrm{O}_{12} \mathrm{U}$

Exact Mass: 1027.11

Molecular Weight: 1029.44

$\mathrm{m} / \mathrm{z}: 1029.11(100.0 \%), 1027.11(51.4 \%), 1031.11(51.4 \%), 1030$

$(16.4 \%), 1031.12(5.1 \%), 1029.12(3.8 \%), 1033.12(2.6 \%), 1033.11(1.4 \%), 1032.12(1.3 \%), 1030.12(1.1 \%)$

Elemental Analysis: C, 33.83; H, 3.43; Br, 15.52; N, 5.44; O, 18.65; U, 23.12

\section{[Bis(Ethyl-a(4-bromophenylazo acetoacetato) diacetato] dioxouranium (VI)}

Figure 3

\section{Antimicrobial studies of Complexes:}

The antimicrobial activities of different representative complex compound samples under studies were tested against different microbial species using Methods reported earlier ${ }^{25-27}$ on nutrient agar NA medium using paper disc method. Results obtained are presented in the table 18,19 given below. None of the complex compound products showed any significant antimicrobial activity

Table 18:Biological Activity Zone of Inhibition for Fungal Growth for Various Complexes of Dioxouranium(VI)

\begin{tabular}{|c|c|c|c|c|c|}
\hline \multirow[t]{2}{*}{ Complexes (20 mg/ml) } & \multicolumn{5}{|c|}{ Zone of Inhibition (mm) } \\
\hline & $\begin{array}{l}\text { Fungal } \\
\text { M.F. }\end{array}$ & SP M.G. & A.N. & P.S. & S.C. \\
\hline Control Cyclohexamide $(10 \mathrm{mg} / \mathrm{ml})$ & 10 & 12 & 16 & 16 & 20 \\
\hline $\begin{array}{l}\text { [Bis (Ethyl- } \alpha-(3-\text { chloro } \\
\text { Dioxouranium (VI) }\end{array}$ & - & - & - & - & - \\
\hline $\begin{array}{l}\text { [Bis (Ethyl- } \alpha-\text { (3-chloro phenylazo) } \\
\text { Dioxouranium (VI) }\end{array}$ & - & - & - & - & - \\
\hline
\end{tabular}

$\mathrm{MF}=$ Microsporum fulvum

$\mathrm{AN}=$ Aspergillus niger

$\mathrm{SC}=$ Saccharomyces cerevisiae
MG= Microsporum gypseum

$\mathrm{PS}=$ Penicillium species

Table 19: Biological Activity Zone of Inhibition for Bacterial Growth for Various Complexes of Dioxouranium (VI)

\begin{tabular}{|c|c|c|c|c|c|}
\hline \multirow[t]{2}{*}{ Complexes $(20 \mathrm{mg} / \mathrm{ml})$} & \multicolumn{5}{|c|}{ Zone of Inhibition (mm) } \\
\hline & $\begin{array}{l}\text { Bacterial } \\
\text { E.A. }\end{array}$ & $\begin{array}{l}\text { SP } \\
\text { P.V. }\end{array}$ & E.C. & S.A. & B.S. \\
\hline Tetracycline $(10 \mathrm{mg} / \mathrm{ml})$ & 20 & 25 & 10 & 32 & 30 \\
\hline $\begin{array}{llll}\text { [Bis } \quad(\text { Ethyl }-\alpha-(3-\text { chloro } & \text { phenylazo }) & \text { acetoacetato }) & \text { diiodo }] \\
\text { Dioxouranium }(V I) & & \end{array}$ & - & 9 & - & 8 & - \\
\hline $\begin{array}{llll}\text { [Bis (Ethyl- } \alpha-(3-\text { chloro } & \text { phenylazo }) & \text { acetoacetato }) & \text { dinitro] } \\
\text { Dioxouranium }(V I) & & \end{array}$ & - & 8 & - & 8 & 9 \\
\hline
\end{tabular}

$\mathrm{EA}=$ Enterobacter aerogens

$\mathrm{EC}=$ Escherichia coil

$\mathrm{BS}=$ Bacillus subtilis

\section{$\mathrm{PV}=$ Proteus vulgaris}

$\mathrm{SA}=$ Staphylococcus aureus 


\section{References}

[1]. Kirby,H.W.; Morss, L.R. Actinium. In The chemistry of the Actinde and Transactinide Elements; Springer: Dordrecht, The Netherlands,; Volume i, pp. 18-51.(2006)

[2]. Cotton S.C. Coordination chemistry of the actinides. In Lanthanide and Actinide Chemistry; john Wiley \& Sons, Ltd.: Sussex, UK,(2006.)

[3]. Jayarami, R.M.; Sudhavani, T.J.' Sivagangi, R. Synergistic extraction of uranium (VI) by complexation with CYANEX-272 and CYANEX-923 TPBD, TNBD, TOPO in presence of nitrate. Int. j.;Res.Chem.Environ.,2,158-163.( 2012)

[4]. Vercouter, T.; Vitorge, P.; Amekraz, B.; Giffaut, E.; Hubert, S.; Moulin, C. Stabilities of the Aqueous Complexes $\left.\mathrm{Cm}_{(\mathrm{CO}}\right)_{3}{ }^{3-}$ and $\mathrm{Am}\left(\mathrm{CO}_{3}\right)_{3}{ }^{3-}$ in the Temperature Range 10-70 ${ }^{\circ} \mathrm{C}$ Inorg.Chem., 5833-5843.( 2005)

[5]. Polinski, M.J.; Wang, S.; Alekseev, E.V.; Depmeier, W.; Liu, G,; Haire, R.G.; Albrecht-schmitt, T.E.Curium(III) borate shows coordination environments of both plutonium(III) and Americium(III) borates. Angew. Chem.Int.Ed, 51, 1869-1872.( 2012)

[6]. K. Arora and Sonal Agnihotri, Reviews in Inorganic Chemistry, Vol. 26, No. 6, pp. 565-593, (2006).

[7]. R.K. Agrawal, H. Agrawal and K. Arora, Rev. Inorg. Chem., 20, 1 (2002)

[8]. S. Agnihotri and Kishor Arora E Journal of chemistry 7 (3) 1045, (2010)

[9]. Mallikarsin S. Yadawe and Sangamesh A. Patil, Global J. Science Frontier Research Chemistry, 12 (2002) ISSN 2249-4626.

[10]. Vaibhav, Raut, S.P. Roya, M.K. Das, S. Jeya kumar. K.L. Ram Kumar, Inter National Journal of analytical Mass Spectrometry and Chromatography, 1, 61-71 (2013).

[11]. V.S. Jolly, M.Y. Dalvi and A.K. Shrivastav, J. Indian Chem, SOC. Vol. 68, pp. 513-514 (1991).

[12]. V.S. Jolly, A.K. Halve and A.K. Shrivastava, Indian J. Chem., 12, 1117 (1978).

[13]. K. Shrivastava and J.K. Malhotra, Indian J. Appl. Chem., 32, 116(1969).

[14]. V.S. Jolly, A.K. Shrivastava, S.P. Sing and K.S. Tiwari, Journal of Indian Chem SOC. Volume 57 (LVII) Page $539-541$ (1980).

[15]. Robert M. Silverstein and Francis X. Webster, Spectrometric Identification of Organic Compounds, $6^{\text {th }}$ Ed., John Wiley \& Sons, Inc., New York, ISBN : 0-471-13457-0, , p. 104.( 1997)

[16]. R.B. King, Inorg. Chem., 5, , p. 300.( 1966 )

[17]. R.B. Agrawal and G.K. Agrawal, J. Indian Chem. Soc., 55, , p. 681.(1978)

[18]. A.B.P. Lever, E. Mautio Veni and B.S. Ramaswamy, Canld. J. Chem., 49, 1957 (1971).

[19]. R.K. Agrawal, K. Arora and Prashant Dutt, Synth. React. Inorg. Met. Org. Chem., 24(2), 301, (1994).

[20]. R.K. Agrawal and K. Arora, Synth. React. Inorg. Met. Org. Chem., 23(10), 1671, (1993).

[21]. R.K. Agrawal, K. Arora, Miss Priyanka and I. Chakravorti, Polish J. Chem., 67, 1913 (1993).

[22]. E.D. Eastman, L. Brown, L.A. Bromley, P.W. Gilles, N.L. Lofgern, J. Am. Chem. Soc., 72, 4019,( 1950)

[23]. R.K. Agarwal, K. Arora and P. Dutt, Polyhedron, 13(6), 957, (1994).

[24]. Sonal Agnihotri and Kishor Arora, Asian J. Chem., 25(8), 4323,( 2013).

[25]. Bauer AW, Kirby WM, Sherris JC, Turck M. Antibiotic susceptibility testing by a standardized single disk method. Am J Clin Pathol. Apr; 45(4), pp. 493-496.( 1966)

[26]. Kishor Arora, D.P. Sharma and Susheel Sharma, Oxozirconium (IV) Complexes with Schiff Bases of -aminopyridine, Asian J. Chem., 11(4), 1357 (1999).

[27]. Kishor Arora, R.C. Goyal, Susheel Sharma and M.C. Pathak, Studies of Dioxouranium (VI) Metal Complexes with a Schiff Base of -aminopyridine, Asian J. Chem., 11(3), 1005 (1999). 